\title{
A pestle and mortar approach for room temperature defect engineering in metal oxides
}

\author{
Yu Huang
}

Impurity doping and defect engineering represent common strategies for tailoring the electronic properties of solid-state materials, for example, controlling the carrier type and carrier concentration in semiconductor materials. More recently, it has been shown that by the introduction of certain impurities the electronic band structure of $\mathrm{TiO}_{2}$ can be greatly modified, resulting in substantially different electronic and optical properties. For example, Chen et al. [1] reported the discovery of a defective "black" $\mathrm{TiO}_{2}$ fabricated by annealing pristine powders in $\mathrm{H}_{2}$ atmosphere (20 bar) at $200^{\circ} \mathrm{C}$ for 5 days. Unlike typical white $\mathrm{TiO}_{2}$ that does not absorb visible light, "black" $\mathrm{TiO}_{2}$ strongly absorbs visible photons and shows extraordinary photocatalytic activity in the visible range, which has generated considerable excitement among the photocatalysis community. Similarly, Nakajima et al. [2] used pulsed laser-induced oxygen release process to produce defective black $\mathrm{TiO}_{2}$ with enhanced $\mathbf{a}$

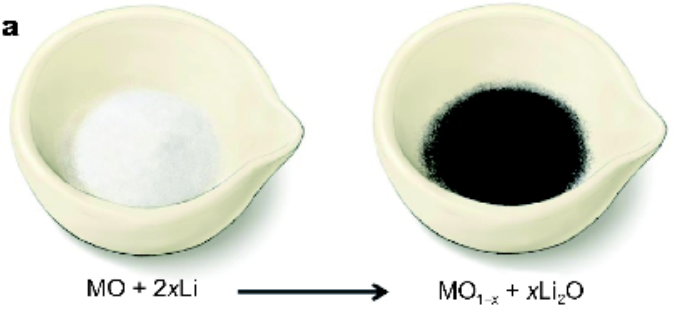

d
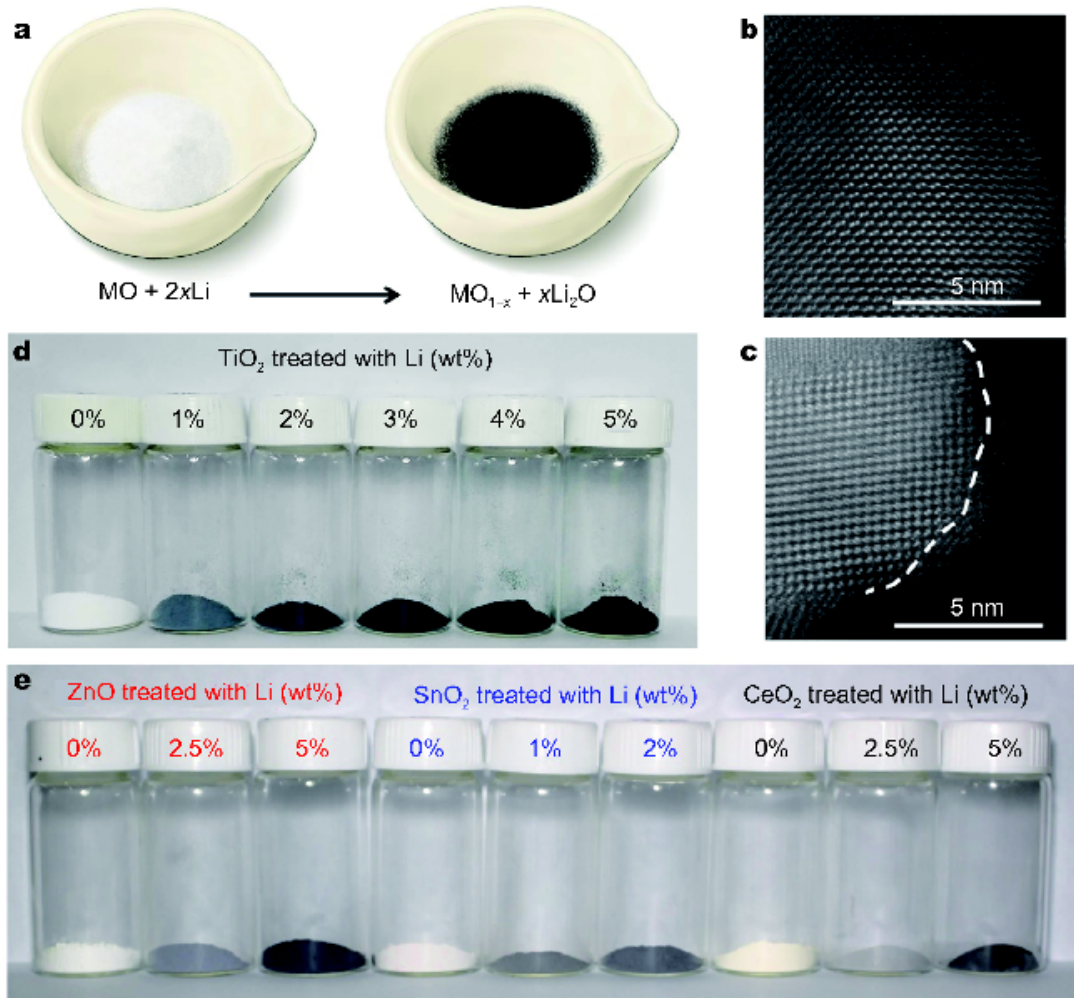

c

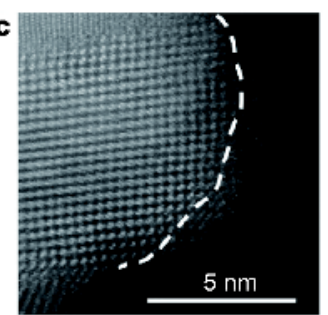

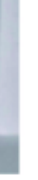

Figure 1 (a) Schematic diagram for defect engineering of oxides by lithium reduction strategy. (b and c) High angle annular dark field (HAADF) images of pristine and $5 \%$ lithium-reduced $\mathrm{TiO}_{2}$. (d) Photograph of $\mathrm{TiO}_{2}$. (e) Photograph of $\mathrm{ZnO}, \mathrm{SnO}_{2}$, and $\mathrm{CeO}$, respectively. Reprinted with permission from Ref. [5], Copyright 2018, Springer Nature.

Department of Materials Science and Engineering, University of California, Los Angeles, CA 90095, USA

Email: yhuang@seas.ucla.edu 
solar water splitting efficiency. In parallel, reductive metals, such as $\mathrm{Mg}$ and $\mathrm{Al}$, have also been introduced to tailor the defects in $\mathrm{TiO}_{2}$ for the production of black $\mathrm{TiO}_{2}$ for visible light-assisted hydrogen production $[3,4]$.

These studies have highlighted the exciting potential in defect engineering of metal oxide for tailoring their electronic, optical and catalytic properties. However, the approaches used to engineer such defects to date usually require some rather complicated procedures involving high-temperature, high pressure and long-duration and highly specialized laser ablation, which are costly and can lead to undesired crystal structural and morphological changes of nanomaterials. Additionally, it remains a significant challenge to precisely control the defects concentration to generate tunable properties.

To these challenges, $\mathrm{Wu}$ and $\mathrm{Li}$ et al. [5] from Tsinghua University and their collaborators including Li-Min Liu from Beihang University reported a new lithium reduction strategy to generate defects in $\mathrm{TiO}_{2}$ nanoparticles to produce black $\mathrm{TiO}_{2}$ at room temperature. With their approach, defective oxide can be readily produced by grinding oxides with Li powders in a mortar (Fig. 1a). Thanks to the high reducibility of $\mathrm{Li}$, it can readily extract oxygen from $\mathrm{TiO}_{2}$, resulting in defects and disorder and producing black $\mathrm{TiO}_{2}$ (Fig. 1b, c). This approach is easy to control, fast, versatile and readily scalable. Notably, the defects in the oxides can be tuned by adjusting the ratio of $\mathrm{Li}$ to oxides, producing $\mathrm{TiO}_{2}$ with different levels of defects and hence different colors (Fig. 1d, e). Their study further shows that these colored $\mathrm{TiO}_{2}$ exhibit excellent photocatalytic activities and stability.

This study defines a facile, rapid and controllable way to engineer the defects in $\mathrm{TiO}_{2}$ for scalable production of "black" $\mathrm{TiO}_{2}$ at room temperature, and may open up exciting opportunities for photocatalyst applications. More broadly, the lithium reduction approach may offer a general pathway for defect engineering and for tailoring the electronic structures of a wide range of metal oxides. According to Ellingham diagrams, most oxides can be reduced by lithium metal at room temperature. Indeed, the authors have shown that their method can be readily extended to implant defects into a series of oxide materials, including $\mathrm{ZnO}, \mathrm{SnO}_{2}$ and $\mathrm{CeO}_{2}$ (Fig. 1e). This approach may be further extended for defect engineering in diverse material systems beyond metal oxides, including sulfides and selenides, opening up a new pathway to tailor and tame the electronic and optical properties of the relevant materials for broad applications in electronics, photonics and catalysis.

Received 20 April 2018; accepted 20 April 2018; published online 17 May 2018

1 Chen X, Liu L, Yu PY, et al. Increasing solar absorption for photocatalysis with black hydrogenated titanium dioxide nanocrystals. Science, 2011, 331: 746-750

2 Nakajima T, Nakamura T, Shinoda K, et al. Rapid formation of black titania photoanodes: pulsed laser-induced oxygen release and enhanced solar water splitting efficiency. J Mater Chem A, 2014, 2: 6762-6771

3 Sinhamahapatra A, Jeon JP, Yu JS. A new approach to prepare highly active and stable black titania for visible light-assisted hydrogen production. Energy Environ Sci, 2015, 8: 3539-3544

4 Wang Z, Yang C, Lin T, et al. Visible-light photocatalytic, solar thermal and photoelectrochemical properties of aluminium-reduced black titania. Energy Environ Sci, 2013, 6: 3007-3014

5 Ou G, Xu Y, Wen B, et al. Tuning defects in oxides at room temperature by lithium reduction. Nat Commun, 2018, 9: 1302 